\title{
Supplementary Appendix
}

Supplement to: Nielsen J, Cunningham SA, Ali MK, Patel SA. Spouse's diabetes and incidence of depression and anxiety: A 20 year prospective study

\section{Contents}

Figure S1. Flowchart: Selection of the Study Population Selection from the Panel Study of Income Dynamics Cohort.

Table S1. Incidence of depression and/or anxiety according to spouse's diabetes status over the period 1999-2017: Additional Adjustment for the study participants own chronic condition status.

Table S2. Incidence rate ratios of depression and/or anxiety according to spouse's diabetes status, stratified by sex. 


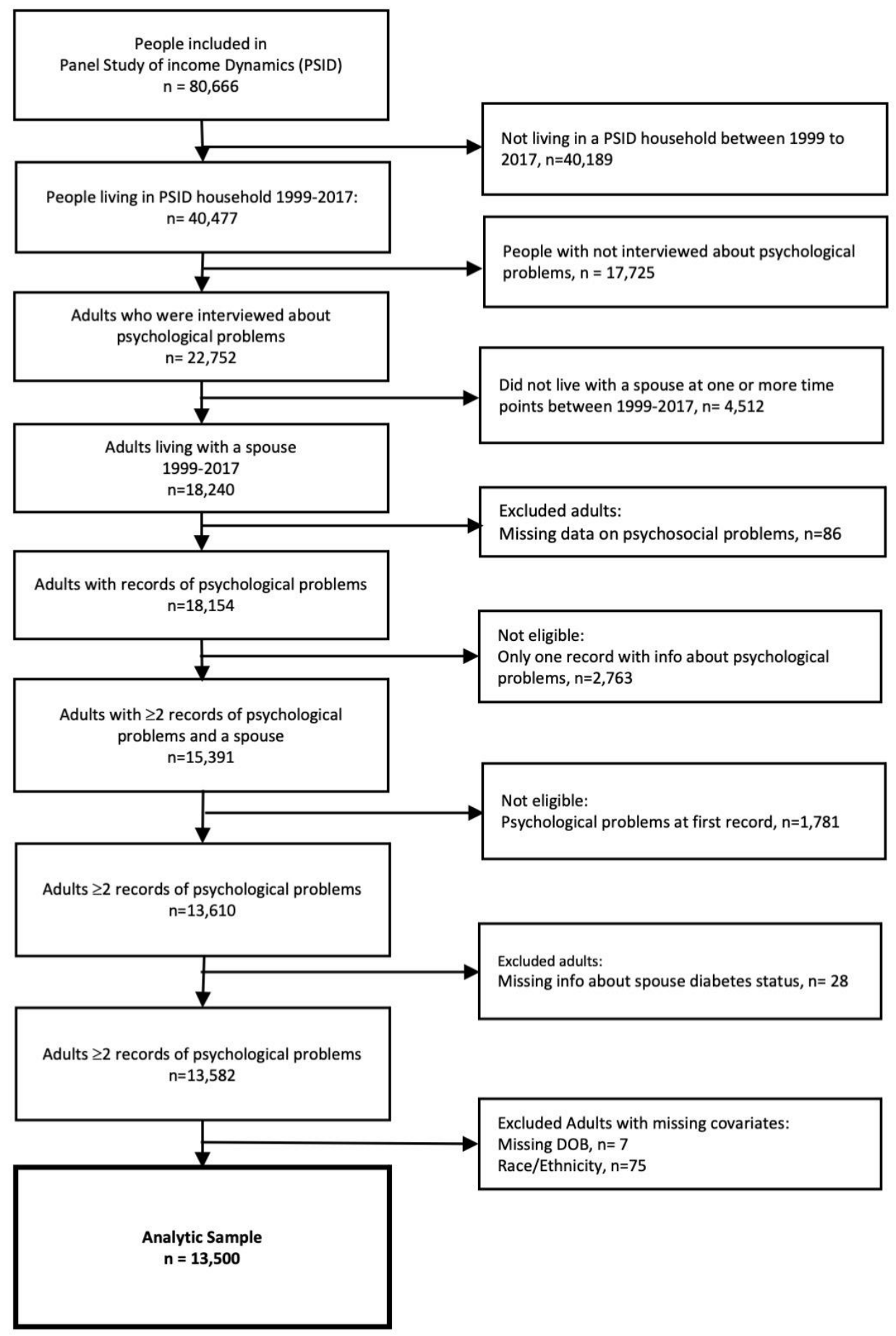

Figure S1. Flowchart: Selection of the Study Population Selection from the Panel Study of Income Dynamics Cohort. 
Table S1. Incidence of depression and/or anxiety according to spouse's diabetes status over the period 1999-2017: Additional Adjustment for the study participants own chronic condition status

\begin{tabular}{|c|c|c|c|}
\hline \multirow{2}{*}{$\begin{array}{l}\text { Spouse's diabetes } \\
\text { status }\end{array}$} & \multicolumn{3}{|c|}{ Incidence rate ratio $(95 \% \mathrm{CI})$} \\
\hline & $\begin{array}{l}\text { Adjustment: } \\
\text { age, sex, race }\end{array}$ & $\begin{array}{c}\text { Adjustment: } \\
+ \text { own diabetes }\end{array}$ & $\begin{array}{c}\text { Adjustment: } \\
+ \text { own other chronic } \\
\text { conditions }\end{array}$ \\
\hline $\begin{array}{l}\text { Spouse has no } \\
\text { diabetes }\end{array}$ & Ref & Ref & Ref \\
\hline $\begin{array}{l}\text { Spouse has } \\
\text { diabetes }\end{array}$ & $1.24(1.01 ; 1.53)$ & $1.19(0.97 ; 1.47)$ & $1.18(0.97 ; 1.45)$ \\
\hline \multicolumn{4}{|l|}{$\begin{array}{l}\text { Diabetes with } \\
\text { limitations }\end{array}$} \\
\hline No diabetes & Ref & Ref & Ref \\
\hline $\begin{array}{l}\text { Diabetes without } \\
\text { limitations }\end{array}$ & $1.07(0.84 ; 1.36)$ & $1.03(0.80 ; 1.31)$ & $1.03(0.81 ; 1.32)$ \\
\hline $\begin{array}{l}\text { Diabetes with } \\
\text { limitations }\end{array}$ & $1.89(1.35 ; 2.67)$ & $1.77(1.26 ; 2.50)$ & $1.66(1.17 ; 2.34)$ \\
\hline \multicolumn{4}{|l|}{$\begin{array}{l}\text { Diabetes and } \\
\text { comorbidities }\end{array}$} \\
\hline $\begin{array}{l}\text { No diabetes no } \\
\text { other diseases }\end{array}$ & Ref & Ref & Ref \\
\hline $\begin{array}{l}\text { No diabetes but } \\
\text { other diseases }\end{array}$ & $2.18(1.85 ; 2.58)$ & $2.16(1.83 ; 2.55)$ & $2.04(1.72 ; 2.41)$ \\
\hline $\begin{array}{l}\text { Diabetes no other } \\
\text { diseases }\end{array}$ & $1.09(0.81 ; 1.47)$ & $1.05(0.78 ; 1.42)$ & $1.07(0.79 ; 1.44)$ \\
\hline $\begin{array}{l}\text { Diabetes and other } \\
\text { diseases }\end{array}$ & $2.34(1.78 ; 3.09)$ & $2.23(1.69 ; 2.64)$ & $2.07(1.56 ; 2.73)$ \\
\hline \multicolumn{4}{|l|}{ Duration $^{1}$} \\
\hline No diabetes & Ref & Ref & Ref \\
\hline Prevalent diabetes & $1.33(1: 01 ; 1.75)$ & $1.27(0: 96 ; 1.67)$ & $1.25(0: 95 ; 1.64)$ \\
\hline Incident diabetes & $1.16(0.87 ; 1.55)$ & $1.11(0.84 ; 1.48)$ & $1.11(0.83 ; 1.48)$ \\
\hline
\end{tabular}


Table S2. Incidence rate ratios of depression and/or anxiety according to spouse's diabetes status, stratified by sex

Spouse's diabetes status

Women

Men

\begin{tabular}{|c|c|c|c|c|c|c|c|c|}
\hline & $\begin{array}{l}\text { Incidence rate } \\
\text { per } 1000 \\
\text { Person-year }\end{array}$ & $\begin{array}{c}\text { IRR } \\
\text { Adjustment } \\
\text { level } 1\end{array}$ & $\begin{array}{c}\text { IRR } \\
\text { Adjustment } \\
\text { level } 2\end{array}$ & $\begin{array}{c}\text { IRR } \\
\text { Adjustment } \\
\text { level } 3\end{array}$ & $\begin{array}{c}\text { Incidence rate } \\
\text { per } 1000 \\
\text { Person-year }\end{array}$ & $\begin{array}{c}\text { IRR } \\
\text { Adjustment } \\
\text { level } 1\end{array}$ & $\begin{array}{c}\text { IRR } \\
\text { Adjustment } \\
\text { level } 2\end{array}$ & $\begin{array}{c}\text { IRR } \\
\text { Adjustment } \\
\text { level } 3\end{array}$ \\
\hline Spouse has no diabetes & $7.7(7.0 ; 8.5)$ & Ref & Ref & Ref & $5.3(4.7 ; 5.9)$ & Ref & Ref & Ref \\
\hline Spouse has diabetes & $8.3(6.0 ; 10.6)$ & $1.07(0.80 ; 1.44)$ & $1.01(0.75 ; 1.36)$ & $0.99(0.73 ; 1.34)$ & $8.1(5.9 ; 10.2)$ & $1.52(1.14 ; 2.03)$ & $1.48(1.10 ; 1.97)$ & $1.42(1.06 ; 1.90)$ \\
\hline
\end{tabular}

Diabetes with limitations

No diabetes

Diabetes without limitations

$7.7(7.0 ; 8.5)$

$6.9(4.6 ; 9.3)$

Ref

Ref

$5.3(4.7 ; 5.9)$

$1.48(1.10 ; 1.97)$

Diabetes with limitations

$13.2(7.0 ; 19.4)$

$0.90(0.63 ; 1.28)$

$0.86(0.60 ; 1.22)$

Ref

Ref

$7.1(4.8 ; 9.3)$

$1.33(0.95 ; 1.87)$

Ref

Ref

$1.71(1.05 ; 2.76) \quad 1.54(0.95 ; 2.50) \quad 1.46(0.90 ; 2.36)$

$11.7(6.2 ; 17.1)$

$2.20(1.36 ; 3.57)$

$1.30(0.92 ; 1.82) \quad 1.26(0.90 ; 1.77)$

Diabetes and comorbidities

No diabetes no other diseases

$7.0(6.2 ; 7.7)$

Ref

Ref

Ref

$4.0(3.4 ; 4.5)$

Ref

$2.12(1.31 ; 3.44)$

$1.98(1.22 ; 3.23)$

No diabetes but other disease

$12.6(9.9 ; 15.4)$

$1.81(1.42 ; 2.32)$

$1.78(1.39 ; 2.28)$

$1.75(1.37 ; 2.24)$

$5.7(3.3 ; 8.2)$

$0.83(0.53 ; 1.28)$

$0.78(0.50 ; 1.20)$

Diabetes and other diseases

$14.3(9.0 ; 19.7)$

$2.06(1.39 ; 3.05)$

$1.93(1.30 ; 2.87)$

$1.87(1.26 ; 2.77)$

$10.8(8.9 ;$

$6.2(3.8 ; 8.7)$

Ref

Ref

Ref

$11.6(7.4 ; 15.8)$

$1.57(1.04 ; 2.37)$

$2.70(2.14 ; 3.40) \quad 2.64(2.09 ; 3.33)$

$1.54(1.02 ; 2.32) \quad 1.49(0.99 ; 2.26)$

Duration $^{1}$

No diabetes

Prevalent diabetes

$7.7(7.0 ; 8.5)$

Ref

Ref

Ref

$5.2(4.7 ; 5.9)$

$9.8(6.5 ; 13.2)$

Ref Ref

$2.81(1.89 ; 4.17)$

$2.67(1.79 ; 3.97)$

$6.2(3.5 ; 8.9)$

Ref

Ref

Values are presented as incidence rate ratios with $95 \%$ confidence intervals.

Adjustment level 1: Age, race/ethnicity

Adjustment level 2: Age, race/ethnicity, own diabetes status

Adjustment level 3: Age, race/ethnicity, household poverty status

${ }^{1}$ Duration of spouse's diabetes: Prevalent diabetes is defined as living with a spouse with diabetes at study entry; Incident diabetes is defined as living with or moving in with a spouse who developed diabetes. 